\title{
Successive Iteration of Positive Solutions for Fourth-Order Two-Point Boundary Value Problems
}

\author{
Yongping Sun, Xiaoping Zhang, and Min Zhao \\ College of Electron and Information, Zhejiang University of Media and Communications, Hangzhou, Zhejiang 310018, China \\ Correspondence should be addressed to Yongping Sun; sunyongping@126.com
}

Received 1 March 2013; Revised 12 June 2013; Accepted 14 June 2013

Academic Editor: Chuanzhi Bai

Copyright (C) 2013 Yongping Sun et al. This is an open access article distributed under the Creative Commons Attribution License, which permits unrestricted use, distribution, and reproduction in any medium, provided the original work is properly cited.

\begin{abstract}
We are concerned with a fourth-order two-point boundary value problem. We prove the existence of positive solutions and establish iterative schemes for approximating the solutions. The interesting point of our method is that the nonlinear term is involved with all lower-order derivatives of unknown function, and the iterative scheme starts off with a known cubic function or the zero function. Finally we give two examples to verify the effectiveness of the main results.
\end{abstract}

\section{Introduction}

The bending of an elastic beam can be described with some fourth-order boundary value problems. Owing to their importance in engineering, physics, and material mechanics, boundary value problems for elastic beam equations have attracted much attention of many authors. Many methods, such as upper and lower solutions method, shooting method, coincidence degree theory, or fixed point theorem and so on, have been developed to derive existence of solutions for fourth-order differential equations with two-point boundary conditions; see [1-20] and many references cited therein. However, almost all of the papers we mentioned focused their attention on the existence of solutions or positive solutions. In the existing literature, there are few papers that concern with the computational methods of solutions or positive solutions; see [21-24]. In this paper, by the successively iterative technique, we study the existence and iteration of monotone positive solutions for the following fourth-order differential equation:

$$
\begin{array}{r}
u^{\prime \prime \prime \prime}(t)=q(t) f\left(t, u(t), u^{\prime}(t), u^{\prime \prime}(t), u^{\prime \prime \prime}(t)\right), \\
\text { a.e. } t \in[0,1],
\end{array}
$$

together with two-point boundary conditions

$$
u(0)=u^{\prime}(1)=u^{\prime \prime}(0)=u^{\prime \prime \prime}(1)=0 .
$$

Throughout this paper, the following conditions are satisfied:

(A1) $f \in C([0,1] \times[0, \infty) \times[0, \infty) \times(-\infty, 0] \times(-\infty, 0]$, $[0, \infty))$;

(A2) $q \in L^{1}[0,1]$ is nonnegative and $0<\int_{0}^{1} q(s) d s<\infty$.

In material mechanics, (1) describes the deflection of a beam under a certain force. The boundary conditions of the forms (2) mean that the elastic beam is simply fixed at the end $t=0$ and fastened with a sliding clamp at the end $t=1$.

Fourth-order differential equations with boundary conditions (2) have been studied. For example, Graef and Yang in [7] studied the nonlinear fourth-order differential equation:

$$
u^{\prime \prime \prime \prime}(t)=\lambda a(t) f(u(t)), \quad 0<t<1,
$$

together with boundary conditions (2). The authors established some sufficient conditions for the existence and nonexistence of positive solutions. The main tool is the Kranosel'skii fixed point theorem. Using the Leggett-Williams fixed point theorem, Yang [18] established an existence criterion for triple positive solutions of the nonlinear fourth-order differential equation:

$$
u^{\prime \prime \prime \prime}(t)=g(t) f\left(t, u(t), u^{\prime}(t)\right), \quad 0<t<1,
$$

with two-point boundary conditions (2). By applying iterative techniques, Sun and Wang [21] investigated the existence 
and iteration of monotone positive solution for the following elastic beam equation

$$
u^{\prime \prime \prime \prime}(t)=f\left(t, u(t), u^{\prime}(t)\right), \quad 0<t<1,
$$

with boundary conditions (2). By using Leray-Schauder nonlinear alternate, Leray-Schauder fixed point theorem and a fixed point theorem due to Avery and Peterson, Sun [15] presented some results on the existence and multiplicity of positive solutions to problem (1)-(2). Bai [3] proposed a constructive method which yields two monotone sequences that converge uniformly to extremal solutions to the following differential equations

$$
\begin{aligned}
& u^{\prime \prime \prime \prime}(t)-M u^{\prime \prime}(t) \\
& \quad=f\left(t, u(t), u^{\prime}(t), u^{\prime \prime}(t), u^{\prime \prime \prime}(t)\right), \quad 0<t<1,
\end{aligned}
$$

or

$$
\begin{aligned}
& u^{\prime \prime \prime \prime}(t)-M u^{\prime \prime}(t) \\
& \quad=f\left(t, u(t), u^{\prime}(t), u^{\prime \prime}(t)\right), \quad 0<t<1,
\end{aligned}
$$

with boundary conditions (2), if there exists an upper solution $\beta$ and a lower solution $\alpha$ with $\beta \leqslant \alpha$. By placing some restrictions on the nonlinear term $f$, Bai [4] obtained the existence results for the fourth-order two-point boundary value problem (1)-(2) via the lower and upper solution method. In particular, a new truncating technique and an appropriate Nagumo-type condition are introduced and employed. In addition, Yao studied (1) with nonhomogeneous boundary condition

$$
u(0)=a, \quad u^{\prime}(1)=b, \quad u^{\prime \prime}(0)=c, \quad u^{\prime \prime \prime}(1)=d .
$$

In [20] the author obtained local existence theorem via LeraySchauder fixed point theorem. In [22] Yao constructed an iterative sequence by the monotonic technique and proved that the sequence approximates successively to the solution of the equation under suitable assumptions.

Motivated by the above-mentioned works, this paper investigates the existence and iteration of positive solutions for problem (1)-(2). It is noted that the successively iterative scheme starts off with the zero function or a simple cubic function, which is feasible for the computational purpose. At the end of the paper, two examples are included to illustrate the main results.

\section{Preliminaries}

In this section, we introduce some necessary definitions and properties of the Green function.

Definition 1 (see $[25,26]$ ). Let $E$ be a real Banach space. A nonempty closed convex set $K \subset E$ is called a cone of $E$ if it satisfies the following two conditions:

(1) $x \in K, \lambda \geqslant 0$ imply $\lambda x \in K$;

(2) $x \in K,-x \in K$ imply $x=0$.
Definition 2 (see $[25,26]$ ). An operator is called completely continuous if it is continuous and maps bounded sets into precompact sets.

The Green's function $G:[0,1] \times[0,1] \rightarrow[0,+\infty)$ for problem (1)-(2) is given by

$$
G(t, s)=\frac{1}{6} \begin{cases}\left(6 t-3 t^{2}-s^{2}\right) s, & 0 \leqslant s \leqslant t \leqslant 1 \\ \left(6 s-3 s^{2}-t^{2}\right) t, & 0 \leqslant t \leqslant s \leqslant 1\end{cases}
$$

so solving the boundary value problem (1)-(2) is equivalent to finding a solution to the integral equation

$$
u(t)=\int_{0}^{1} G(t, s) q(s) f\left(s, u(s), u^{\prime}(s), u^{\prime \prime}(s), u^{\prime \prime \prime}(s)\right) d s
$$

Some properties of functions $G(t, s)$ are summarized as follows.

Lemma 3. For all $t, s \in[0,1]$, the following inequalities hold true:

$$
\begin{array}{cc}
0 \leqslant-\frac{\partial^{3} G(t, s)}{\partial t^{3}} \leqslant 1, & 0 \leqslant-\frac{\partial^{2} G(t, s)}{\partial t^{2}} \leqslant t \\
0 \leqslant \frac{\partial G(t, s)}{\partial t} \leqslant \frac{1}{2}\left(1-t^{2}\right), & 0 \leqslant G(t, s) \leqslant \frac{1}{6}\left(3 t-t^{3}\right) .
\end{array}
$$

Proof. For any fixed $s \in[0,1]$, from (9) we obtain

$$
\begin{gathered}
\frac{\partial^{3} G(t, s)}{\partial t^{3}}= \begin{cases}0, & 0 \leqslant s<t \leqslant 1, \\
-1, & 0 \leqslant t<s \leqslant 1,\end{cases} \\
\frac{\partial^{2} G(t, s)}{\partial t^{2}}= \begin{cases}-s, & 0 \leqslant s \leqslant t \leqslant 1, \\
-t, & 0 \leqslant t \leqslant s \leqslant 1,\end{cases}
\end{gathered}
$$

then,

$$
0 \leqslant-\frac{\partial^{3} G(t, s)}{\partial t^{3}} \leqslant 1, \quad 0 \leqslant-\frac{\partial^{2} G(t, s)}{\partial t^{2}} \leqslant t, \quad t, s \in[0,1] .
$$

For all $t, s \in[0,1]$, it follows from (9) that

$$
\begin{aligned}
0 & \leqslant \frac{\partial G(t, s)}{\partial t} \\
& =\left\{\begin{array}{ll}
(1-t) s, & 0 \leqslant s \leqslant t \leqslant 1, \\
s-\frac{1}{2} s^{2}-\frac{1}{2} t^{2}, & 0 \leqslant t \leqslant s \leqslant 1,
\end{array} \leqslant \frac{1}{2}\left(1-t^{2}\right) .\right.
\end{aligned}
$$

For all $t, s \in[0,1]$, if $s \leqslant t$, it yields

$$
\begin{aligned}
G(t, s) & =\frac{1}{6}\left(6 t-3 t^{2}-s^{2}\right) s=\frac{1}{6}\left[3-3(1-t)^{2}-s^{2}\right] s \\
& \leqslant \frac{1}{6}\left(3-s^{2}\right) s \leqslant \frac{1}{6}\left(3 t-t^{3}\right) .
\end{aligned}
$$


While $t \leqslant s$, we have

$$
\begin{aligned}
G(t, s) & =\frac{1}{6}\left(6 s-3 s^{2}-t^{2}\right) t=\frac{1}{6}\left[3-3(1-s)^{2}-t^{2}\right] t \\
& \leqslant \frac{1}{6}\left(3 t-t^{3}\right) .
\end{aligned}
$$

The proof is completed.

In what follows we will consider the Banach space $E=$ $C^{3}[0,1]$ equipped with norm

$$
\begin{gathered}
\|u\|=\max \left\{\max _{0 \leqslant t \leqslant 1}|u(t)|, \max _{0 \leqslant t \leqslant 1}\left|u^{\prime}(t)\right|, \max _{0 \leqslant t \leqslant 1}\left|u^{\prime \prime}(t)\right|,\right. \\
\left.\max _{0 \leqslant t \leqslant 1}\left|u^{\prime \prime \prime}(t)\right|\right\} .
\end{gathered}
$$

Define the cone $K \subset C^{3}[0,1]$ by

$$
\begin{gathered}
K=\left\{u \in C^{3}[0,1]: u(t) \geqslant 0, u^{\prime}(t) \geqslant 0, u^{\prime \prime}(t) \leqslant 0,\right. \\
\left.u^{\prime \prime \prime}(t) \leqslant 0, t \in[0,1]\right\} .
\end{gathered}
$$

For $u \in K$, we give an operator $T$ by

$$
\begin{aligned}
& (\mathrm{Tu})(t) \\
& =\int_{0}^{1} G(t, s) q(s) f\left(s, u(s), u^{\prime}(s), u^{\prime \prime}(s), u^{\prime \prime \prime}(s)\right) d s .
\end{aligned}
$$

Obviously, the fixed points of $T$ are monotone and nonnegative solutions of the problem (1)-(2).

Lemma 4. Consider that $T: K \rightarrow K$ is completely continuous.

Proof. Suppose that $u \in K$. Using (A1), (A2), (19), and Lemma 3, we obtain $(T u)(t) \geqslant 0,(T u)^{\prime}(t) \geqslant 0,(T u)^{\prime \prime}(t) \leqslant 0$, $(T u)^{\prime \prime \prime}(t) \leqslant 0$, for all $t \in[0,1]$. Then $T(K) \subseteq K$. Using arguments similar to those of $[21,22]$, one can prove that $T$ is a completely continuous operator.

\section{Main Results}

In this section, we give the main results of this paper.

Theorem 5. Assume that there exists $R>0$ such that

(H1) $f\left(t, x_{1}, y_{1}, z_{1}, w_{1}\right) \leqslant f\left(t, x_{2}, y_{2}, z_{2}, w_{2}\right)$ for any $0 \leqslant t \leqslant$ $1,0 \leqslant x_{1} \leqslant x_{2} \leqslant R, 0 \leqslant y_{1} \leqslant y_{2} \leqslant R, 0 \leqslant-z_{1} \leqslant-z_{2} \leqslant$ $R, 0 \leqslant-w_{1} \leqslant-w_{2} \leqslant R$;

(H2) $\max _{0 \leqslant t \leqslant 1} f(t, R, R,-R,-R) \leqslant \Lambda R$, where $\Lambda=$ $\left(\int_{0}^{1} q(s) d s\right)^{-1}$

(H3) $f(t, 0,0,0,0) \not \equiv 0$.

Then problem (1)-(2) has two monotone positive solutions $w^{*}$ and $v^{*}$ satisfying $\left\|w^{*}\right\| \leqslant R,\left\|v^{*}\right\| \leqslant R$ and $\lim _{n \rightarrow \infty} \| w_{n}$ $w^{*}\left\|=0, \lim _{n \rightarrow \infty}\right\| v_{n}-v^{*} \|=0$, where $w_{n}=T^{n} w_{0}, v_{n}=T^{n} v_{0}$, $n=1,2, \ldots, w_{0}(t)=(R / 6)\left(3 t-t^{3}\right)$, and $v_{0}(t)=0$, for $t \in$ $[0,1]$.
Proof. Let $K_{R}=\{u \in K:\|u\| \leqslant R\}$; it follows that $T: K_{R} \rightarrow$ $K_{R}$. In fact, if $u \in K_{R}$, then $\|u\| \leqslant R$; thus, for any $t \in[0,1]$,

$$
\begin{gathered}
0 \leqslant u(t) \leqslant \max _{0 \leqslant t \leqslant 1} u(t) \leqslant\|u\| \leqslant R, \\
0 \leqslant u^{\prime}(t) \leqslant \max _{0 \leqslant t \leqslant 1} u^{\prime}(t) \leqslant\|u\| \leqslant R, \\
0 \leqslant-u^{\prime \prime}(t) \leqslant \max _{0 \leqslant t \leqslant 1}\left(-u^{\prime \prime}(t)\right) \leqslant\|u\| \leqslant R, \\
0 \leqslant-u^{\prime \prime \prime}(t) \leqslant \max _{0 \leqslant t \leqslant 1}\left(-u^{\prime \prime \prime}(t)\right) \leqslant\|u\| \leqslant R,
\end{gathered}
$$

which together with the conditions $(H 1)$ and $(H 2)$ implies that

$$
\begin{aligned}
& 0 \leqslant(T u)(t) \\
& =\int_{0}^{1} G(t, s) q(s) f\left(s, u(s), u^{\prime}(s), u^{\prime \prime}(s), u^{\prime \prime \prime}(s)\right) d s \\
& \leqslant \frac{1}{6}\left(3 t-t^{3}\right) \int_{0}^{1} q(s) f(s, R, R,-R,-R) d s \\
& \leqslant \frac{1}{2} \Lambda R \int_{0}^{1} q(s) d s=\frac{1}{2} R, \quad t \in[0,1], \\
& 0 \leqslant(T u)^{\prime}(t) \\
& =\int_{0}^{1} \frac{\partial G(t, s)}{\partial t} q(s) f\left(s, u(s), u^{\prime}(s), u^{\prime \prime}(s), u^{\prime \prime \prime}(s)\right) d s \\
& \leqslant \frac{1}{2}\left(1-t^{2}\right) \int_{0}^{1} q(s) f(s, R, R,-R,-R) d s \\
& \leqslant \frac{1}{2} \Lambda R \int_{0}^{1} q(s) d s=\frac{1}{2} R, \quad t \in[0,1], \\
& 0 \leqslant-(T u)^{\prime \prime}(t) \\
& =-\int_{0}^{1} \frac{\partial^{2} G(t, s)}{\partial t^{2}} q(s) f\left(s, u(s), u^{\prime}(s), u^{\prime \prime}(s), u^{\prime \prime \prime}(s)\right) d s \\
& \leqslant t \int_{0}^{1} q(s) f(s, R, R,-R,-R) d s \\
& \leqslant \Lambda R \int_{0}^{1} q(s) d s=R, \quad t \in[0,1] \\
& 0 \leqslant-(T u)^{\prime \prime \prime}(t) \\
& =-\int_{0}^{1} \frac{\partial^{3} G(t, s)}{\partial t^{3}} q(s) f\left(s, u(s), u^{\prime}(s), u^{\prime \prime}(s), u^{\prime \prime \prime}(s)\right) d s \\
& \leqslant \int_{0}^{1} q(s) f(s, R, R,-R,-R) d s \\
& \leqslant \Lambda R \int_{0}^{1} q(s) d s=R, \quad t \in[0,1]
\end{aligned}
$$

Hence, we have shown that $\|T u\| \leqslant R$, that is, $T: K_{R} \rightarrow K_{R}$.

(1) Let $w_{0}(t)=(R / 6)\left(3 t-t^{3}\right), t \in[0,1]$; then $\left\|w_{0}\right\|=$ $R$ and $w_{0} \in K_{R}$. Denote $w_{n}=T^{n} w_{0}, n=1,2,3, \ldots$. Since 
$T: K_{R} \rightarrow K_{R}$, we have $w_{n} \in T\left(K_{R}\right) \subseteq K_{R}, n=1,2,3, \ldots$ Since $T$ is completely continuous, we assert that $\left\{w_{n}\right\}_{n=1}^{\infty}$ is a sequentially compact set.

Since $w_{1}=T w_{0} \in K_{R}$, by Lemma 3, (H1), and (H2), we obtain

$w_{1}(t)$

$=\left(T w_{0}\right)(t)$

$=\int_{0}^{1} G(t, s) q(s) f\left(s, w_{0}(s), w_{0}^{\prime}(s), w_{0}^{\prime \prime}(s), w_{0}^{\prime \prime \prime}(s)\right) d s$

$=\int_{0}^{1} G(t, s) q(s) f\left(s, \frac{R}{6}\left(3 s-s^{3}\right)\right.$,

$$
\left.\frac{R}{2}\left(1-s^{2}\right),-R s,-R\right) d s
$$

$\leqslant \frac{1}{6}\left(3 t-t^{3}\right) \int_{0}^{1} q(s) f(s, R, R,-R,-R) d s$

$\leqslant \frac{R}{6}\left(3 t-t^{3}\right) \Lambda \int_{0}^{1} q(s) d s=\frac{R}{6}\left(3 t-t^{3}\right)=w_{0}(t)$,

$t \in[0,1]$,

$w_{1}^{\prime}(t)$

$=\left(T w_{0}\right)^{\prime}(t)$

$=\int_{0}^{1} \frac{\partial G(t, s)}{\partial t} q(s) f\left(s, w_{0}(s), w_{0}^{\prime}(s), w_{0}^{\prime \prime}(s), w_{0}^{\prime \prime \prime}(s)\right) d s$

$=\int_{0}^{1} \frac{\partial G(t, s)}{\partial t} q(s) f\left(s, \frac{R}{6}\left(3 s-s^{3}\right)\right.$,

$$
\left.\frac{R}{2}\left(1-s^{2}\right),-R s,-R\right) d s
$$

$\leqslant \frac{1}{2}\left(1-t^{2}\right) \int_{0}^{1} q(s) f(s, R, R,-R,-R) d s$

$\leqslant \frac{R}{2}\left(1-t^{2}\right) \Lambda \int_{0}^{1} q(s) d s=\frac{R}{2}\left(1-t^{2}\right)=w_{0}^{\prime}(t)$,

$t \in[0,1]$,

$$
\begin{aligned}
& -w_{1}^{\prime \prime}(t) \\
& =-\left(T w_{0}\right)^{\prime \prime}(t) \\
& =-\int_{0}^{1} \frac{\partial^{2} G(t, s)}{\partial t^{2}} q(s) f\left(s, w_{0}(s), w_{0}^{\prime}(s), w_{0}^{\prime \prime}(s), w_{0}^{\prime \prime \prime}(s)\right) d s \\
& =-\int_{0}^{1} \frac{\partial^{2} G(t, s)}{\partial t^{2}} q(s) f\left(s, \frac{R}{6}\left(3 s-s^{3}\right),\right. \\
& \left.\frac{R}{2}\left(1-s^{2}\right),-R s,-R\right) d s
\end{aligned}
$$

$$
\begin{aligned}
& \leqslant t \int_{0}^{1} q(s) f(s, R, R,-R,-R) d s \\
& \leqslant t \Lambda R \int_{0}^{1} q(s) d s=R t=-w_{0}^{\prime \prime}(t), \quad t \in[0,1], \\
& -w_{1}^{\prime \prime \prime}(t) \\
& =-\left(T w_{0}\right)^{\prime \prime \prime}(t) \\
& =-\int_{0}^{1} \frac{\partial^{3} G(t, s)}{\partial t^{3}} q(s) f\left(s, w_{0}(s), w_{0}^{\prime}(s), w_{0}^{\prime \prime}(s), w_{0}^{\prime \prime \prime}(s)\right) d s \\
& =-\int_{0}^{1} \frac{\partial^{3} G(t, s)}{\partial t^{3}} q(s) f\left(s, \frac{R}{6}\left(3 s-s^{3}\right),\right. \\
& \leqslant \int_{0}^{1} q(s) f(s, R, R,-R,-R) d s \\
& \leqslant \Lambda R \int_{0}^{1} q(s) d s=R=-w_{0}^{\prime \prime \prime}(t), \quad t \in[0,1] .
\end{aligned}
$$

Thus,

$$
\begin{aligned}
& w_{1}(t) \leqslant w_{0}(t), \quad w_{1}^{\prime}(t) \leqslant w_{0}^{\prime}(t), \quad-w_{1}^{\prime \prime}(t) \leqslant-w_{0}^{\prime \prime}(t), \\
& -w_{1}^{\prime \prime \prime}(t) \leqslant-w_{0}^{\prime \prime \prime}(t), \quad t \in[0,1] .
\end{aligned}
$$

Furthermore, it follows from $(H 1)$ that

$$
\begin{aligned}
w_{2}(t)=\left(T w_{1}\right)(t) & \leqslant\left(T w_{0}\right)(t)=w_{1}(t), \quad t \in[0,1], \\
w_{2}^{\prime}(t)=\left(T w_{1}\right)^{\prime}(t) & \leqslant\left(T w_{0}\right)^{\prime}(t)=w_{1}^{\prime}(t), \quad t \in[0,1] \\
-w_{2}^{\prime \prime}(t) & =-\left(T w_{1}\right)^{\prime \prime}(t) \leqslant-\left(T w_{0}\right)^{\prime \prime}(t) \\
& =-w_{1}^{\prime \prime}(t), \quad t \in[0,1], \\
-w_{2}^{\prime \prime \prime}(t) & =-\left(T w_{1}\right)^{\prime \prime \prime}(t) \leqslant-\left(T w_{0}\right)^{\prime \prime \prime}(t) \\
& =-w_{1}^{\prime \prime \prime}(t), \quad t \in[0,1] .
\end{aligned}
$$

By the induction, we obtain

$$
\begin{array}{r}
w_{n+1}(t) \leqslant w_{n}(t), \quad w_{n+1}^{\prime}(t) \leqslant w_{n}^{\prime}(t), \\
-w_{n+1}^{\prime \prime}(t) \leqslant-w_{n}^{\prime \prime}(t), \quad-w_{n+1}^{\prime \prime \prime}(t) \leqslant-w_{n}^{\prime \prime \prime}(t), \\
t \in[0,1], n=0,1,2, \ldots .
\end{array}
$$

Hence, there exists $w^{*} \in K_{R}$ such that $\lim _{n \rightarrow \infty}\left\|w_{n}-w^{*}\right\|=0$. Applying the continuity of $T$ and using $w_{n+1}=T w_{n}$, we obtain $T w^{*}=w^{*}$, which implies that $w^{*}$ is a nonnegative solution of problem (1)-(2). Furthermore, by (H3) we know that the zero function is not a solution of problem (1)-(2). Thus, $\max _{0 \leqslant t \leqslant 1} w^{*}(t)>0$. From the definition of the cone $K$, 
it yields $w^{*}(t) \geqslant t\left\|w^{*}\right\|>0,\left(w^{*}\right)^{\prime}(t) \geqslant 0, t \in(0,1)$; that is, $w^{*}(t)$ is a monotone positive solution of problem (1)-(2).

(2) Let $v_{0}(t)=0, t \in[0,1]$; then $v_{0} \in K_{R}$. Let $v_{n}=$ $T^{n} v_{0}, n=1,2,3, \ldots$. With respect to $T: K_{R} \rightarrow K_{R}$, we obtain $v_{n} \in T\left(K_{R}\right) \subseteq K_{R}, n=1,2,3, \ldots$. Since $T$ is completely continuous, we assert that $\left\{v_{n}\right\}_{n=1}^{\infty}$ is a sequentially compact set.

$$
\begin{aligned}
\text { Since } v_{1} & =T v_{0}=T 0 \in K_{R}, \text { we obtain } \\
R \geqslant v_{1}(t) & =\left(T v_{0}\right)(t)=(T 0)(t) \geqslant 0=v_{0}(t), \quad t \in[0,1], \\
R \geqslant v_{1}^{\prime}(t)= & \left(T v_{0}\right)^{\prime}(t)=(T 0)^{\prime}(t) \geqslant 0=v_{0}^{\prime}(t), \quad t \in[0,1], \\
R & \geqslant-v_{1}^{\prime \prime}(t)=-\left(T v_{0}\right)^{\prime \prime}(t)=-(T 0)^{\prime \prime}(t) \\
& \geqslant 0=-v_{0}^{\prime \prime}(t), \quad t \in[0,1], \\
R & \geqslant-v_{1}^{\prime \prime \prime}(t)=-\left(T v_{0}\right)^{\prime \prime \prime}(t)=-(T 0)^{\prime \prime \prime}(t) \\
& \geqslant 0=-v_{0}^{\prime \prime \prime}(t), \quad t \in[0,1] .
\end{aligned}
$$

So by $(H 1)$, we have

$$
\begin{aligned}
v_{2}(t)=\left(T v_{1}\right)(t) \geqslant\left(T v_{0}\right)(t)=v_{1}(t), \quad t \in[0,1] \\
v_{2}^{\prime}(t)=\left(T v_{1}\right)^{\prime}(t) \geqslant\left(T v_{0}\right)^{\prime}(t)=v_{1}^{\prime}(t), \quad t \in[0,1], \\
-v_{2}^{\prime \prime}(t)=-\left(T v_{1}\right)^{\prime \prime}(t) \geqslant-\left(T v_{0}\right)^{\prime \prime}(t) \\
=-v_{1}^{\prime \prime}(t), \quad t \in[0,1], \\
-v_{2}^{\prime \prime \prime}(t)=-\left(T v_{1}\right)^{\prime \prime \prime}(t) \geqslant-\left(T v_{0}\right)^{\prime \prime \prime}(t) \\
=-v_{1}^{\prime \prime \prime}(t), \quad t \in[0,1] .
\end{aligned}
$$

By the induction, we further obtain

$$
\begin{aligned}
& v_{n+1}(t) \geqslant v_{n}(t), \quad v_{n+1}^{\prime}(t) \geqslant v_{n}^{\prime}(t), \quad-v_{n+1}^{\prime \prime}(t) \geqslant-v_{n}^{\prime \prime}(t), \\
& -v_{n+1}^{\prime \prime \prime}(t) \geqslant-v_{n}^{\prime \prime \prime}(t), \quad t \in[0,1], \quad n=0,1,2, \ldots
\end{aligned}
$$

Hence, there exists $v^{*} \in K_{R}$ such that $\lim _{n \rightarrow \infty}\left\|v_{n}-v^{*}\right\|=0$, and $v^{*}$ is a monotone positive solution of problem (1)-(2). The proof is completed.

Corollary 6. Suppose that $\left(H_{1}\right)$ and $\left(H_{3}\right)$ hold. Assume

$$
\text { (H4) } \lim _{x \rightarrow+\infty} \max _{0 \leqslant t \leqslant 1} f(t, x, x,-x,-x) / x<\Lambda \text {. }
$$

Then problem (1)-(2) has at least two monotone positive solutions $w^{*}$ and $v^{*}$ satisfying the conclusion of Theorem 5 .

Remark 7. $w^{*}$ and $v^{*}$ may coincide with each other and then the problem (1)-(2) has only one monotone positive solution in $K_{R}$.
Remark 8. By the proof of Theorem $5,\left\{w_{n}\right\}_{n=1}^{\infty}$ and $\left\{v_{n}\right\}_{n=1}^{\infty}$ converge in $C^{3}[0,1]$ to the monotone positive solution $w^{*}$ and $v^{*}$ of the problem (1)-(2), respectively. In addition, the sequences $\left\{w_{n}\right\}_{n=1}^{\infty},\left\{v_{n}\right\}_{n=1}^{\infty},\left\{w_{n}^{\prime}\right\}_{n=1}^{\infty}$, and $\left\{v_{n}^{\prime}\right\}_{n=1}^{\infty}$ are increasing, while the sequences $\left\{w_{n}^{\prime \prime}\right\}_{n=1}^{\infty},\left\{v_{n}^{\prime \prime}\right\}_{n=1}^{\infty},\left\{w_{n}^{\prime \prime \prime}\right\}_{n=1}^{\infty}$, and $\left\{v_{n}^{\prime \prime \prime}\right\}_{n=1}^{\infty}$ are decreasing.

Remark 9. The iterative schemes in Theorem 5 start off with a known cubic function or the zero function, which is feasible for the computational purpose.

\section{Examples}

In this section we provide two examples to illustrate the application of our results.

Example 1. Consider the problem

$$
\begin{aligned}
u^{\prime \prime \prime \prime}(t)= & \frac{1}{2} t+\frac{1}{18} u^{2}(t)+\frac{1}{6} u^{\prime}(t)-\frac{1}{6} u^{\prime \prime}(t) \\
& -\frac{1}{6} u^{\prime \prime \prime}(t), \quad t \in[0,1] \\
u(0)= & u^{\prime}(1)=u^{\prime \prime}(0)=u^{\prime \prime \prime}(1)=0
\end{aligned}
$$

For this problem, $f(t, x, y, z, w)=t+(1 / 9) x^{2}+(1 / 3) y-$ $(1 / 3) z-(1 / 3) w$ and $q(t)=1 / 2$. By the calculations, the conditions (A1), (A2), and (H1)-(H3) of Theorem 5 hold for $R=6$. From Theorem 5, we have that problem (29) has two monotone positive solutions $w^{*}$ and $v^{*}$ satisfying $\left\|w^{*}\right\| \leqslant$ 6, $\left\|v^{*}\right\| \leqslant 6$ and $\lim _{n \rightarrow \infty}\left\|w_{n}-w^{*}\right\|=0, \lim _{n \rightarrow \infty}\left\|v_{n}-v^{*}\right\|=$ 0 .

Moreover, the two iterative schemes are

$$
w_{0}(t)=3 t-t^{3}, \quad t \in[0,1]
$$

$$
\begin{gathered}
w_{n+1}(t) \\
=\frac{1}{12} \int_{0}^{t}(t-s)^{3} \\
\quad \times\left(s+\frac{1}{9} w_{n}^{2}(s)+\frac{1}{3} w_{n}^{\prime}(s)-\frac{1}{3} w_{n}^{\prime \prime}(s)-\frac{1}{3} w_{n}^{\prime \prime \prime}(s)\right) d s \\
+\frac{t}{12} \int_{0}^{1}\left(6 s-3 s^{2}-t^{2}\right) \\
\quad \times\left(s+\frac{1}{9} w_{n}^{2}(s)+\frac{1}{3} w_{n}^{\prime}(s)-\frac{1}{3} w_{n}^{\prime \prime}(s)\right. \\
\left.-\frac{1}{3} w_{n}^{\prime \prime \prime}(s)\right) d s, \quad t \in[0,1], n=0,1,2, \ldots, \\
v_{0}(t)=0, \quad t \in[0,1],
\end{gathered}
$$




$$
\begin{aligned}
& v_{n+1}(t)= \frac{1}{12} \int_{0}^{t}(t-s)^{3} \\
& \times\left(s+\frac{1}{9} v_{n}^{2}(s)+\frac{1}{3} v_{n}^{\prime}(s)-\frac{1}{3} v_{n}^{\prime \prime}(s)-\frac{1}{3} v_{n}^{\prime \prime \prime}(s)\right) d s \\
&+\frac{t}{12} \int_{0}^{1}\left(6 s-3 s^{2}-t^{2}\right) \\
& \times\left(s+\frac{1}{9} v_{n}^{2}(s)+\frac{1}{3} v_{n}^{\prime}(s)-\frac{1}{3} v_{n}^{\prime \prime}(s)\right. \\
&\left.-\frac{1}{3} v_{n}^{\prime \prime \prime}(s)\right) d s, \\
& t \in[0,1], n=0,1,2, \ldots
\end{aligned}
$$

Using Matlab 7.5, the second and third terms of the two schemes are as follows:

$$
\begin{aligned}
& w_{1}(t)=\frac{3559}{4536} t-\frac{2761}{7560} t^{3}+\frac{1}{16} t^{4}+\frac{1}{80} t^{5}-\frac{1}{5040} t^{8} \\
& +\frac{1}{90720} t^{10}, \quad t \in[0,1] \\
& w_{2}(t)=\frac{5}{48} t-\frac{33627935}{1111065984} t^{3}-\frac{134954777}{11110659840} t^{5} \\
& +\frac{28810105}{8888527872} t^{6}+\frac{75364613}{9258883200} t^{7} \\
& -\frac{22814857}{7407106560} t^{8}-\frac{31759981}{24690355200} t^{9} \\
& +\frac{3292141}{4115059200} t^{10}-\frac{1777}{32659200} t^{11} \\
& -\frac{735019}{35554111488} t^{12}+\frac{48893}{49380710400} t^{13} \\
& -\frac{1227799}{888852787200} t^{14}+\frac{280289}{444426393600} t^{15} \\
& +\frac{10099}{148142131200} t^{16}-\frac{223}{5290790400} t^{17} \\
& +\frac{1}{1567641600} t^{18}+\frac{19}{98761420800} t^{19} \\
& +\frac{1}{10973491200} t^{20}+\frac{19}{444426393600} t^{21} \\
& -\frac{1}{98761420800} t^{22}-\frac{1}{888852787200} t^{23} \\
& +\frac{1}{3555411148800} t^{24}, \quad t \in[0,1],
\end{aligned}
$$

$$
\begin{gathered}
v_{1}(t)=\frac{5}{48} t-\frac{1}{24} t^{3}+\frac{1}{240} t^{5}, \quad t \in[0,1], \\
v_{2}(t)=\frac{5}{48} t-\frac{5159}{124416} t^{3}+\frac{4859}{1244160} t^{5}+\frac{25}{995328} t^{6} \\
+\frac{1}{7776} t^{7}-\frac{5}{248832} t^{8}-\frac{19}{622080} t^{9} \\
+\frac{1}{165888} t^{10}+\frac{11}{3110400} t^{11}-\frac{1}{1244160} t^{12} \\
\quad-\frac{1}{6220800} t^{13}+\frac{1}{24883200} t^{14}, \quad t \in[0,1]
\end{gathered}
$$

Example 2 (see [22]). Consider the problem

$$
\begin{aligned}
u^{\prime \prime \prime \prime}(t)= & \frac{\min \{t, 1-t\}}{48 \sqrt{|1-2 t|}} \\
& \times\left\{2+\left[u(t)+u^{\prime}(t)\right]^{3}-\left[u^{\prime \prime}(t)+u^{\prime \prime \prime}(t)\right]^{3}\right\}, \\
& t \in\left[0, \frac{1}{2}\right) \bigcup\left(\frac{1}{2}, 1\right], \\
& u(0)=u^{\prime}(1)=u^{\prime \prime}(0)=u^{\prime \prime \prime}(1)=0 .
\end{aligned}
$$

In this problem, $f(t, x, y, z, w)=(1 / 48)\left[2+(x+y)^{3}-(z+w)^{3}\right]$ and $q(t)=(\min \{t, 1-t\}) / \sqrt{|1-2 t|}$. Obviously, $q(t)$ and $f(t, x, y, z, w)$ satisfy conditions $(A 1)$ and $(A 2)$. In addition, $f(t, x, y, z, w)$ is increasing with regard to $x$ and $y$ and is decreasing with $z$ and $w$, and $f(t, 0,0,0,0) \not \equiv 0$. Moreover,

$$
\begin{gathered}
\max _{0 \leqslant t \leqslant 1} f\left(t, \frac{1}{30}, \frac{1}{30},-\frac{1}{30},-\frac{1}{30}\right)=\frac{1}{48}\left[2+\frac{2}{3375}\right]<\frac{3}{2} \times \frac{1}{30}, \\
\int_{0}^{1} q(t) d t=\int_{0}^{1} \frac{\min \{t, 1-t\}}{\sqrt{|1-2 t|}} d t=\frac{2}{3} .
\end{gathered}
$$

Therefore, assumptions $(H 1)-(H 3)$ of Theorem 5 are satisfied with $R=1 / 30$. From Theorem 5, problem (32) has two monotone positive solutions $w^{*}$ and $v^{*}$ satisfying $\left\|w^{*}\right\| \leqslant$ $1 / 30,\left\|v^{*}\right\| \leqslant 1 / 30$ and $\lim _{n \rightarrow \infty}\left\|w_{n}-w^{*}\right\|=0, \lim _{n \rightarrow \infty} \| v_{n}-$ $v^{*} \|=0$.

Moreover, the two iterative schemes are

$$
\begin{aligned}
& w_{0}(t)=\frac{1}{180}\left(3 t-t^{3}\right), \quad t \in[0,1], \\
& w_{n+1}(t) \\
& =\frac{1}{144} \int_{0}^{t}(t-s)^{3} \frac{\min \{s, 1-s\}}{\sqrt{|1-2 s|}} \\
& \quad \times\left\{2+\left[w_{n}(s)+w_{n}^{\prime}(s)\right]^{3}\right. \\
& \left.\quad-\left[w_{n}^{\prime \prime}(s)+w_{n}^{\prime \prime \prime}(s)\right]^{3}\right\} d s
\end{aligned}
$$




$$
\begin{aligned}
& +\frac{t}{144} \int_{0}^{1}\left(6 s-3 s^{2}-t^{2}\right) \frac{\min \{s, 1-s\}}{\sqrt{|1-2 s|}} \\
& \times\left\{\left[2+w_{n}(s)+w_{n}^{\prime}(s)\right]^{3}\right. \\
& \left.-\left[w_{n}^{\prime \prime}(s)+w_{n}^{\prime \prime \prime}(s)\right]^{3}\right\} d s, \\
& t \in[0,1], n=0,1,2, \ldots \text {, } \\
& v_{0}(t)=0, \quad t \in[0,1], \\
& v_{n+1}(t) \\
& =\frac{1}{144} \int_{0}^{t}(t-s)^{3} \frac{\min \{s, 1-s\}}{\sqrt{|1-2 s|}} \\
& \times\left\{2+\left[v_{n}(s)+v_{n}^{\prime}(s)\right]^{3}\right. \\
& \left.-\left[v_{n}^{\prime \prime}(s)+v_{n}^{\prime \prime \prime}(s)\right]^{3}\right\} d s \\
& +\frac{t}{144} \int_{0}^{1}\left(6 s-3 s^{2}-t^{2}\right) \frac{\min \{s, 1-s\}}{\sqrt{|1-2 s|}} \\
& \times\left\{2+\left[v_{n}(s)+v_{n}^{\prime}(s)\right]^{3}\right. \\
& \left.-\left[v_{n}^{\prime \prime}(s)+v_{n}^{\prime \prime \prime}(s)\right]^{3}\right\} d s, \\
& t \in[0,1], n=0,1,2, \ldots
\end{aligned}
$$

Using Mathlab 7.5, the second terms of the two schemes are given as follows:

$$
\begin{aligned}
& w_{1}(t) \\
& =-\frac{8449074379661}{23951656146600000}+\frac{441468132942481}{19516164267600000} t \\
& -\frac{10842948935033}{1951616426760000} t^{2}-\frac{785738110303}{169705776240000} t^{3} \\
& +\left(\frac{8449074379661}{23951656146600000}+\frac{122149319510627}{60221306882880000} t\right. \\
& +\frac{2602305979889593}{702581913633600000} t^{2}-\frac{265540119476783}{150553267207200000} t^{3} \\
& \quad-\frac{13518333667229}{19161324917280000} t^{4}-\frac{1855160149}{117096985605600000} t^{5} \\
& \quad-\frac{74353511}{17564547840840000} t^{6}-\frac{26984453}{175645478408400000} t^{7} \\
& +\frac{1291391}{117096985605600000} t^{8}-\frac{4131727}{38322649834560000} t^{9}
\end{aligned}
$$

$$
\begin{aligned}
& -\frac{31823}{2105640100800000} t^{10}+\frac{3953}{491316023520000} t^{11} \\
& \left.+\frac{509}{216757069200000} t^{12}+\frac{1}{5704133400000} t^{13}\right) \\
& \times \sqrt{1-2 t}, \quad \text { for } t \in\left[0, \frac{1}{2}\right] \text {, } \\
& w_{1}(t) \\
& =-\frac{8449074379661}{23951656146600000}+\frac{441468132942481}{19516164267600000} t \\
& -\frac{10842948935033}{1951616426760000} t^{2}-\frac{785738110303}{169705776240000} t^{3} \\
& +\left(-\frac{929396364017203}{2107745740900800000}+\frac{62633084321047}{22910279792400000} t\right. \\
& -\frac{817863130365013}{140516382726720000} t^{2}+\frac{2416385432114987}{526936435225200000} t^{3} \\
& -\frac{10620690488597}{15055326720720000} t^{4}-\frac{568554781}{117096985605600000} t^{5} \\
& -\frac{686043443}{175645478408400000} t^{6}-\frac{546781}{3054703972320000} t^{7} \\
& +\frac{46120147}{234193971211200000} t^{8}-\frac{1594511}{19161324917280000} t^{9} \\
& -\frac{23767}{867028276800000} t^{10}+\frac{11287}{2456580117600000} t^{11} \\
& \left.+\frac{13}{6193059120000} t^{12}+\frac{1}{5704133400000} t^{13}\right) \\
& \times \sqrt{2 t-1}, \quad \text { for } t \in\left[\frac{1}{2}, 1\right] \text {, } \\
& v_{1}(t)=-\frac{1}{2835}+\frac{19}{840} t-\frac{1}{180} t^{2}-\frac{1}{216} t^{3} \\
& +\left(\frac{1}{2835}-\frac{23}{11340} t+\frac{1}{270} t^{2}-\frac{1}{576} t^{3}\right. \\
& \left.-\frac{2}{2835} t^{4}\right) \sqrt{1-2 t}, \quad \text { for } t \in\left[0, \frac{1}{2}\right] \text {, } \\
& v_{1}(t)=-\frac{1}{2835}+\frac{19}{840} t-\frac{1}{180} t^{2}-\frac{1}{216} t^{3} \\
& +\left(-\frac{1}{2268}+\frac{31}{11340} t-\frac{11}{1890} t^{2}+\frac{13}{2835} t^{3}\right. \\
& \left.-\frac{2}{2835} t^{4}\right) \sqrt{2 t-1}, \quad \text { for } t \in\left[\frac{1}{2}, 1\right] \text {. }
\end{aligned}
$$

Remark 10. This example was carried out firstly in [22], in which the author showed that the solution $u^{*} \in C^{3}[0,1]$ is nontrivial, $\left\|u^{*}\right\| \leqslant 1$, and the iterative scheme starts off with

$$
u_{0}(t)=-\frac{7}{24} \int_{0}^{1} G(t, s) \frac{\min \{s, 1-s\}}{\sqrt{|1-2 s|}} d s .
$$

In the present paper, we proved that the solution $u^{*} \in C^{3}[0,1]$ is monotone positive, $\left\|u^{*}\right\| \leqslant 1 / 30$, and the iterative scheme starts off with $u_{0}(t)=(1 / 180)\left(3 t-t^{3}\right)$ or $u_{0}(t)=0$, which is much more simple than that of [22]. 


\section{Acknowledgments}

The authors would like to thank the reviewer for his/her careful reading, suggestions, and comments which improved the presentation of this paper. This work is supported by the Natural Science Foundation of Zhejiang Province of China (LY12A01012).

\section{References}

[1] R. P. Agarwal, "On fourth order boundary value problems arising in beam analysis," Differential and Integral Equations, vol. 2, no. 1, pp. 91-110, 1989.

[2] R. P. Agarwal, D. O'Regan, and P. J. Y. Wong, Positive Solutions of Differential, Difference and Integral Equations, Kluwer Academic, Boston, Mass, USA, 1999.

[3] Z. B. Bai, "Iterative solutions for some fourth-order differential equations," Journal of Systems Science and Mathematical Sciences, vol. 27, no. 4, pp. 555-562, 2007 (Chinese).

[4] Z. Bai, "The upper and lower solution method for some fourthorder boundary value problems," Nonlinear Analysis: Theory, Methods \& Applications, vol. 67, no. 6, pp. 1704-1709, 2007.

[5] Z. Bai and H. Wang, "On positive solutions of some nonlinear fourth-order beam equations," Journal of Mathematical Analysis and Applications, vol. 270, no. 2, pp. 357-368, 2002.

[6] J. Ehme, P. W. Eloe, and J. Henderson, "Upper and lower solution methods for fully nonlinear boundary value problems," Journal of Differential Equations, vol. 180, no. 1, pp. 51-64, 2002.

[7] J. R. Graef and B. Yang, "Existence and nonexistence of positive solutions of fourth order nonlinear boundary value problems," Applicable Analysis, vol. 74, no. 1-2, pp. 201-214, 2000.

[8] J. R. Graef and B. Yang, "Positive solutions of a nonlinear fourth order boundary value problem," Communications on Applied Nonlinear Analysis, vol. 14, no. 1, pp. 61-73, 2007.

[9] Y. Li, "Positive solutions of fourth-order boundary value problems with two parameters," Journal of Mathematical Analysis and Applications, vol. 281, no. 2, pp. 477-484, 2003.

[10] Y. Li, "Two-parameter nonresonance condition for the existence of fourth-order boundary value problems," Journal of Mathematical Analysis and Applications, vol. 308, no. 1, pp. 121-128, 2005.

[11] B. Liu, "Positive solutions of fourth-order two point boundary value problems," Applied Mathematics and Computation, vol. 148, no. 2, pp. 407-420, 2004.

[12] M. Ruyun, Z. Jihui, and F. Shengmao, "The method of lower and upper solutions for fourth-order two-point boundary value problems," Journal of Mathematical Analysis and Applications, vol. 215, no. 2, pp. 415-422, 1997.

[13] R. Ma and H. Wang, "On the existence of positive solutions of fourth-order ordinary differential equations," Applicable Analysis, vol. 59, no. 1-4, pp. 225-231, 1995.

[14] Y. P. Sun, "Symmetric positive solutions to a fourth-order nonlinear differential equation with nonlocal boundary conditions," Acta Mathematica Sinica, vol. 50, no. 3, pp. 547-556, 2007 (Chinese).

[15] Y. P. Sun, "Existence and multiplicity of positive solutions for an elastic beam equation," Applied Mathematics-A Journal of Chinese Universities, vol. 26, no. 3, pp. 253-264, 2011.

[16] B. Yang, "Positive solutions for the beam equation under certain boundary conditions," Electronic Journal of Differential Equations, no. 78, pp. 1-8, 2005.
[17] B. Yang, "Positive solutions for a fourth order boundary value problem," Electronic Journal of Qualitative Theory of Differential Equations, no. 3, pp. 1-17, 2005.

[18] Y. R. Yang, "Triple positive solutions of a class of fourth-order two-point boundary value problems," Applied Mathematics Letters, vol. 23, no. 4, pp. 366-370, 2010.

[19] Q. Yao, "Local existence of multiple positive solutions to a singular cantilever beam equation," Journal of Mathematical Analysis and Applications, vol. 363, no. 1, pp. 138-154, 2010.

[20] Q. Yao, "Solvability of a classical beam equation with Carathéodory nonlinearity," Southeast Asian Bulletin of Mathematics, vol. 35, no. 4, pp. 709-715, 2011.

[21] J. P. Sun and X. Q. Wang, "Existence and iteration of monotone positive solution of BVP for an elastic beam equation," Mathematical Problems in Engineering, vol. 2011, Article ID 705740, 10 pages, 2011.

[22] Q. Yao, "Successively iterative technique of a classical elastic beam equation with Carathéodory nonlinearity," Acta Applicandae Mathematicae, vol. 108, no. 2, pp. 385-394, 2009.

[23] Q. Yao, "Monotonically iterative method of nonlinear cantilever beam equations," Applied Mathematics and Computation, vol. 205, no. 1, pp. 432-437, 2008.

[24] X. Zhang, "Existence and iteration of monotone positive solutions for an elastic beam equation with a corner," Nonlinear Analysis: Real World Applications, vol. 10, no. 4, pp. 2097-2103, 2009.

[25] D. J. Guo and V. Lakshmikantham, Nonlinear Problems in Abstract Cones, vol. 5 of Notes and Reports in Mathematics in Science and Engineering, Academic Press, San Diego, Calif, USA, 1988.

[26] M. A. Krasnosel'skiu, Positive Solutions of Operator Equations, P. Noordhoff, Groningen, The Netherlands, 1964. 


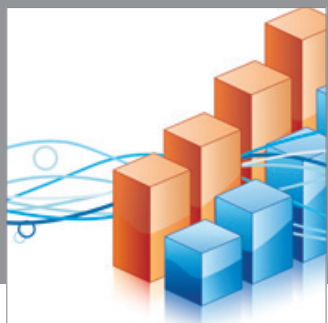

Advances in

Operations Research

mansans

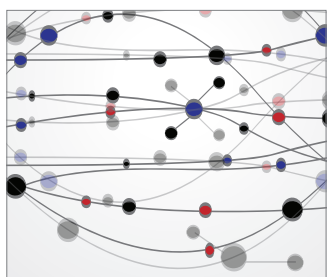

The Scientific World Journal
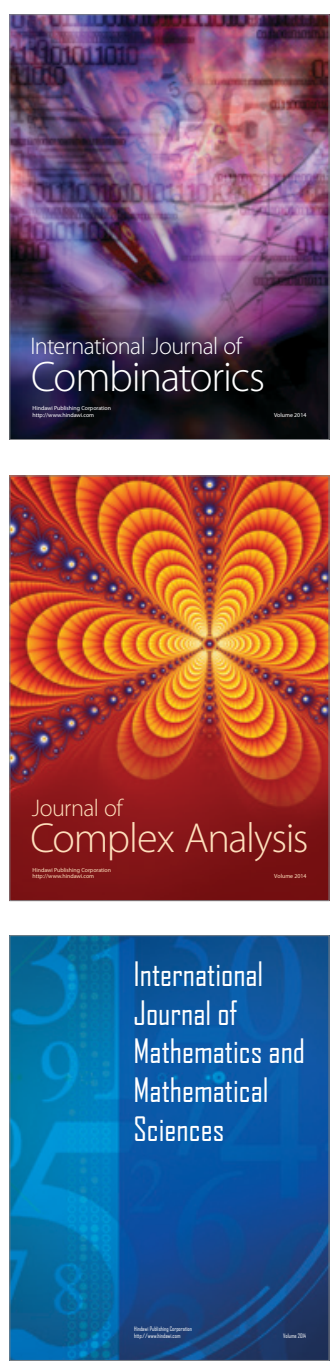
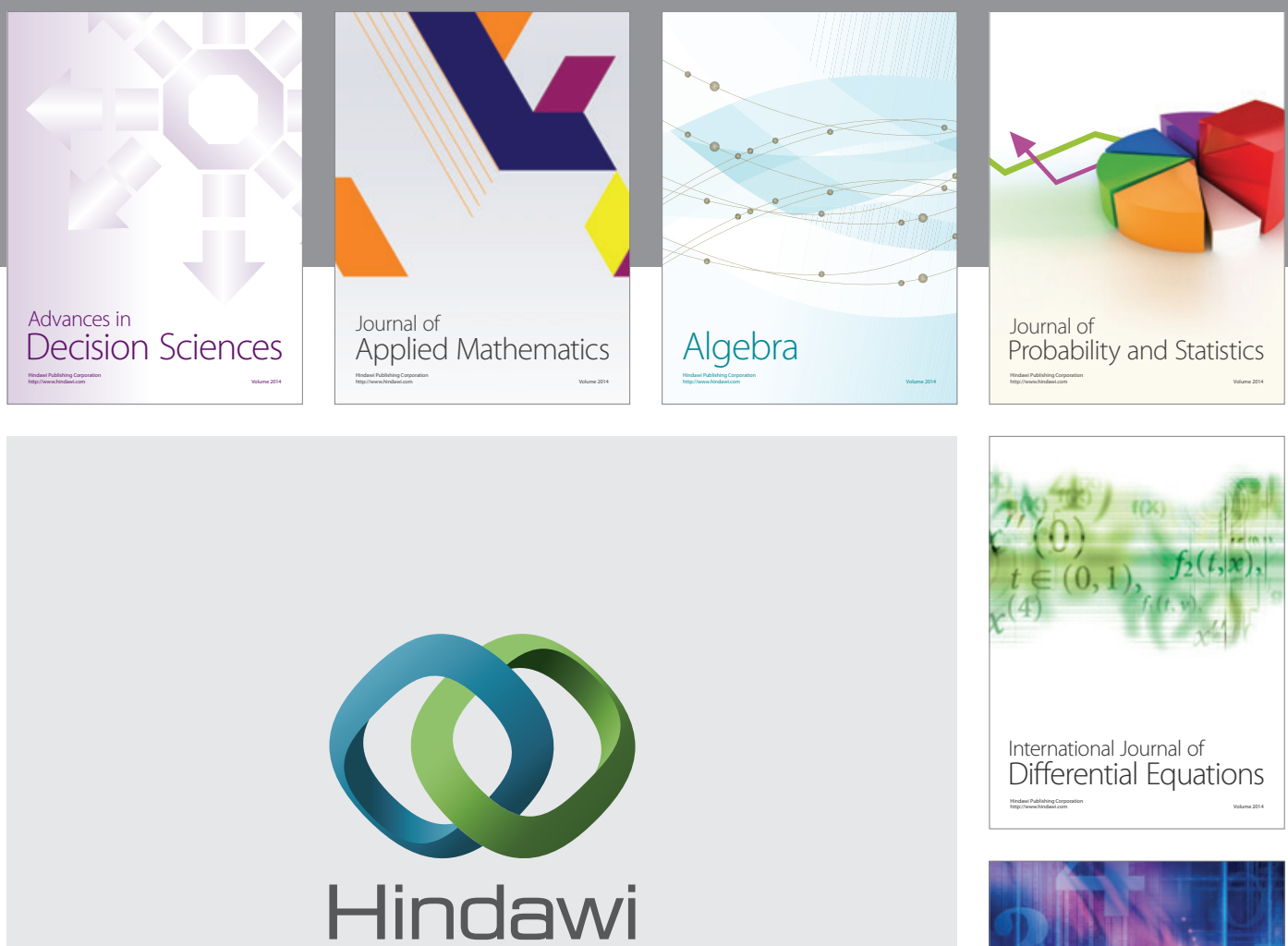

Submit your manuscripts at http://www.hindawi.com
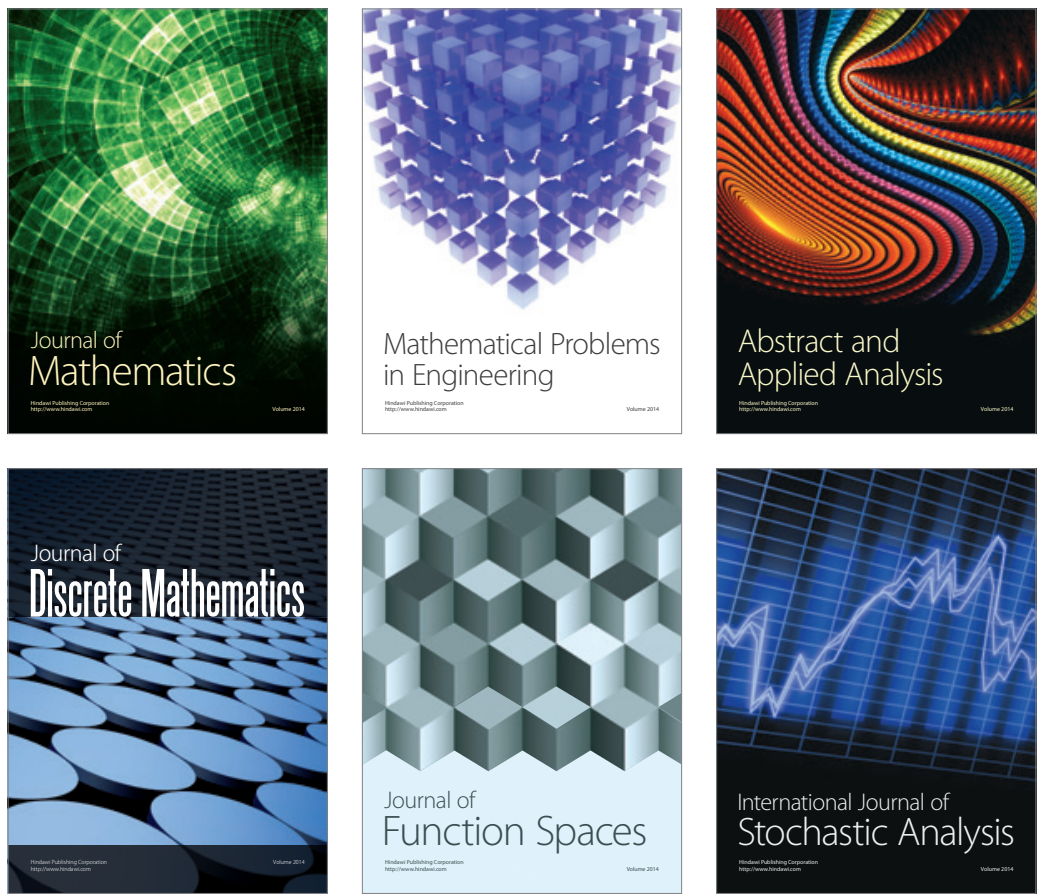

Journal of

Function Spaces

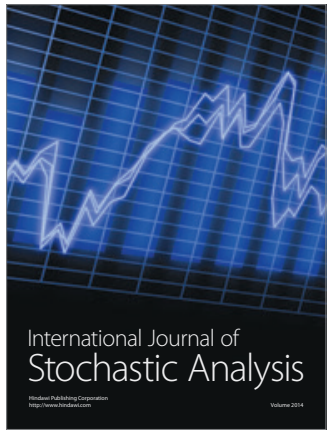

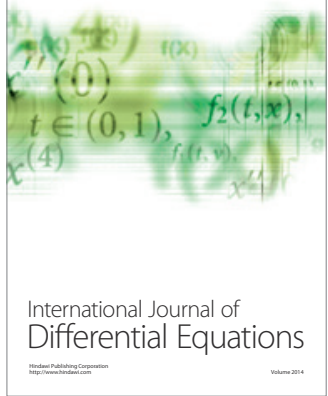
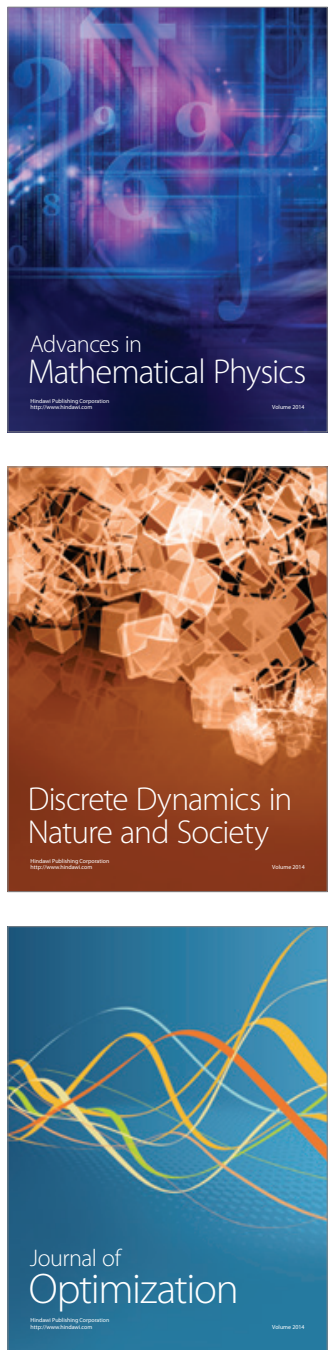